\title{
Neuropatía hereditaria sensitivo- autonómica. Evolución y complicaciones Reporte de un caso
}

Arriaga-Rivera Javier*, Alejo-González Mónica Patricia**, Espinosa-Mejía Martha Janeth***, Arriaga-Rivera Alejandro****

- Resumen

- Las neuropatías hereditarias sensitivas y autonómicas

- (HSAN), son un grupo de enfermedades raras que suelen

- afectar las fibras nerviosas mielínicas y amielínicas, compro-

- metiendo la sensibilidad superficial, profunda y funciones

- autonómicas que se manifiestan en amputaciones, úlceras

- por presión, fracturas, arreflexia, osteomielitis y mutilaciones,

- que conducen a discapacidad, baja calidad de vida y mal

- pronóstico. Los estudios moleculares son el estándar de oro

- para el diagnóstico, sin embargo la electromiografía es un

- estudio confiable que evidencia la afección sensitiva, convir-

- tiéndose en una alternativa como apoyo al diagnóstico en

- nuestro medio. En el caso reportado el desconocimiento de

- la patología condujo a una discapacidad severa, aislamien-

- to social y las múltiples complicaciones que produjeron su

- muerte. LUXMÉDICA AÑ011, NÚMERO 34, SEPTIEMBRE-DICIEMBRE

2016, PP 41-49

Palabras clave: neuropatía, sensitivo-autonómica, complicaciones, discapacidad, calidad de vida.

\begin{abstract}
Hereditary sensory and automatic neuropathy are a group of rare diseases that tend to affect the nerve fibers, myelinated and unmyelinated, compromising superficial and deep sensitivity and autonomic functions that are manifested in amputations, ulcers by pressure, fractures, areflexia, osteomyelitis and mutilation, which lead to disability, low quality of life and poor prognosis. Molecular studies are the standard gold for the diagnosis, however the electromyography is a reliable test that evidences the sensitive condition, becoming in an alternative as support to the diagnosis. In the reported case the ignorance of the disease led to a severe disability, social isolation, and multiple complications that caused his death. LUXMÉDICA AÑ011, NÚMER0 34, SEPTIEMBREDICIEMBRE 2016, PP 41-49

Key words: neuropathy, sensory-autonomic, complications, disability, quality of life.
\end{abstract}

\section{Introducción}

Las neuropatías hereditarias sensitivas-autonómicas (HSAN) son un grupo de enfermedades raras, caracterizadas por alteraciones sensoriales y autonómicas, clasificados en cinco tipos (algunos autores consideran 6) ${ }^{1,2}$, dependiendo de la herencia, neuropatología y síntomas.

* Médico Especialista en Rehabilitación Pediátrica, Subdirector de Clínica del Centro de Rehabilitación Infantil Teletón, Guerrero.

** Médico Especialista en Rehabilitación y Electrodiagnóstico, Subdirector médico operativo del Centro de Rehabilitación Infantil Teletón, Guerrero.

*** Director médico del Centro de Rehabilitación Infantil Teletón, Guerrero. Especialista en Rehabilitación pulmonar.

**** Residente de Medicina de Rehabilitación. Centro de Rehabilitación Infantil Teletón, Guerrero

Fecha de recibido: 18 de julio 2016

Fecha de aceptación: 13 de agosto 2016

Correspondencia: Dr. Javier Arriaga Rivera. Centro de Rehabilitación Infantil Teletón. Boulevard de las Naciones N²607. Colonia Antigua Hacienda el Potrero, Acapulco de Juárez, Guerrero, México, Código postal 39906. Teléfono: 017444341500 Correo electrónico: arriaga@teleton-gro.org.mx 
Cada neuropatía es producida por defectos genéticos específicos que afectan el desarrollo de fibras nerviosas, resultando en una expresión fenotípica variable. El tipo II puede tener un origen autosómico recesivo o presentarse en forma esporádica, con una mutación en el gen WNK1, FAM134B o KIF1A que se localiza en el cromosoma 12p p $^{13.33}$, (OMIM 201300). Suele afectar la sensibilidad superficial y profunda por compromiso de fibras largas tipo A, B y pequeñas tipo $C^{1-3}$ (tabla1).

En la literatura consultada se identificaron escasos reportes considerando su baja prevalencia de 1 en 100000 2,4,5. En nuestro país se encontró un reporte de tres familias afectadas en el estado de San Luis Potosí ${ }^{2}$. A continuación, presentamos un caso detectado en el estado de Guerrero el cual fue atendido en el Centro de Rehabilitación e Inclusión Infantil Teletón a partir de los 12 años de edad. A su ingreso presentaba complicaciones severas, resaltando por tal motivo la necesidad de una intervención oportuna, así como el manejo conductual y educación a la familia para la mejora la calidad de vida.

| | | | | | | | | | | | | | | | | | | | | | | | | | | | | | | | | | | | | | | | | | | | | | | | | | | | | | | | | | | | | | | | | | | | | | | | | | | | | | | | | | | | | | | | | | | | | | | | | | | | | | | | | | |

\section{Presentación del caso clínico}

Antecedentes

Femenino producto de la gesta 1, de madre de 19 años y padre de 31, negando consanguinidad y consumo o abuso de sustancias tóxicas. Embarazo no planeado, sin control prenatal, ni consumo de ácido fólico o hierro, con antecedente de cervicovaginitis al cuarto mes de gestación. Resuelto a las 40 semanas de gestación (SDG) vía cesárea por presentación pélvica, peso de 2850 gramos, Apgar desconocido. Presenta síndrome de dificultad respiratoria, manejándose sólo con $\mathrm{O}_{2}$ suplementario con casco cefálico y se mantuvo en cunero patológico durante cinco días sin presentar complicaciones.

\section{Evolución de la enfermedad y complicaciones}

3 meses: inicia con hipotonía, hipertermia y crisis convulsivas secundarias a hidrocefalia, ameritando colocación de válvula de derivación ventrículoperitoneal, con remisión de las crisis.

6 meses: se agregan lesiones en cavidad oral tipo aftas y asociado a la dentición se presenta una mutilación parcial de lengua.
9 meses: presenta cuadro de conjuntivitis de repetición, dermo-abrasiones en párpados, además de úlceras en ambas córneas.

12 meses: se detecta retraso en la adquisición de los hitos del desarrollo, no logra control cefálico, tronco, gateo, bipedestación o marcha y presenta dificultad para la manipulación fina de objetos, mientras que el desarrollo auditivo y del lenguaje se mantiene en rangos de nor- 
malidad. El tono muscular y los reflejos de estiramiento se reportan disminuidos, por lo que ante estos datos se inicia estudio de probable neuropatía. En relación a la deglución no se identificaron problemas, sin embargo, se dio manejo por antecedente de reflujo gastroesofágico.

4 años: persiste con lesiones tipo úlcera por presión y dermo-abrasiones diseminadas en todo el cuerpo de predominio distal y lesiones por automutilación en falanges distales de ambas manos, contracturas en miembros inferiores (isquiotibiales y tríceps sural). Inicio de bipedestación y marcha a esta edad con dificultad, ameritando apoyo de terceras personas para lograr deambular.

5 años: en cavidad oral se identifica atrofia de papilas gustativas y fístula en paladar que amerita injerto, se toma biopsia del tejido descartando malignidad. En relación a su conducta, se incrementaron los lapsos de autoagresión, dificultando el apego a las indicaciones y un fracaso en la resolución de las lesiones en piel y orientación de la patología.

12 años: acude al Centro de Rehabilitación e Inclusión Infantil Teletón por un cuadro de discapacidad múltiple (visual, motora, cognitiva), donde se integran los siguientes datos por parte del equipo multidisciplinario:

Se realizó una valoración inicial de la medida de independencia funcional versión pediátrica Functional Independence Measure (Wee-FIM por sus siglas en inglés), con un puntaje total de 58/126, con repercusión en las tres áreas que evalúa (cuidado personal, movilidad y cognición), con mayor impacto en la de movilidad, condicionando mayor dependencia para las actividades de vida diaria y aislamiento social ( figura 1).

Se corroboró la hipotonía y arreflexia, mientras que la fuerza por grupos musculares en escala de Lovett fue de 4/5 para miembros superiores y $3 / 5$ para miembros inferiores. Se encontraron contracturas principalmente isquiotibiales de $90^{\circ}$, tríceps sural $10^{\circ}$ y en flexores de caderas en $30^{\circ}$. La sensibilidad superficial y profunda en todas sus variantes se reportó ausente en todas las zonas del cuerpo. Se corroboró una amputación múltiple de falanges distales, excepto del quinto dedo de mano izquierda y amputación del primer dedo de pie izquierdo, además de una úlcera de cuatro $\mathrm{cm}$ de diámetro a nivel de patela derecha, estadio III y escoliosis dorso-lumbar. La electroneuromiografía (ENMG) demostró una polineuropatía sensorial del tipo degeneración axonal en las cuatro extremidades, mientras que las conducciones motoras se encontraron en parámetros normales para todos los nervios evaluados (figura 2). Los potenciales somatosensoriales se reportaron con un bloqueo de la respuesta en nervio tibial y mediano. El estado mental fue abordado como un trastorno del comportamiento con alteraciones en juicio, ánimo y baja tolerancia a la frustración por lo que se inició manejo con risperidona a dosis de 1 mg cada 24 horas, con mejoría parcial de los síntomas. El coeficiente intelectual fue reportado como limítrofe.

13 años: presenta fractura y desplazamiento epifisiario tipo 1 en muñeca derecha (clasificación de Smith) secundaria a caída, ameritando manejo conservador, sin lograrse reducir, produciendo deformidad y edema de tejidos blandos.

14 años: acompañado del edema en miembro torácico derecho, se agrega una lesión tipo úlcera estadio IV a nivel de codo, con destrucción de la articulación, además de otra lesión en pie derecho estadio IV, la cual se complica con una osteomielitis y necrosis de huesos del tarso, desarrollando una sepsis y muerte de la paciente (imágenes 1-3). 


\section{Discusión}

\section{Abordaje diagnóstico}

Los estudios moleculares son el estándar de oro para el diagnóstico de estas patologías $2,6,7$ sin embargo, son estudios costosos y con una baja disponibilidad en países en vías de desarrollo, por lo que se han propuesto 5 tipos de HNSA, que presentan características clínicas específicas, de las cuales podemos apoyarnos para realizar un diagnóstico clínico, tomando en consideración la gran variabilidad del cuadro (véase tabla 2), 4,8 mientras que el diagnóstico diferencial debe contemplar entidades, por ejemplo con otras formas de HSNA, el síndrome de Lesch-Nyhan, fenilcetonuria no tratada, neuropatía diabética y lepra. ${ }^{5}$

El abordaje diagnóstico de este caso se realizó con los datos clínicos y se complementó con el estudio de ENMG, donde se hizo evidente una disminución en la amplitud de los potenciales de acción nerviosos sensoriales (PANS), mientras que los potenciales de acción muscular compuesto (PAMC) fueron normales. La exploración con electrodo de aguja reveló fibrilaciones, elementos que se han reportado en otros estudios con HSAN tipo II. ${ }^{3}$ Cabe aclarar que en forma anatómica las HSNA producen una depleción de las fibras nerviosas sensoriales y autonómicas, por lo que el tamaño de las raíces nerviosas dorsales y los ganglios pueden tener una reducción en su tamaño mayor al $10 \%$. Este dato se corroboró con el bloqueo de la conducción de los potenciales somatosensoriales de nervio tibial y mediano.

Un auxiliar más en el estudio de estas patologías es valorar la respuesta simpática de la piel, ya que es una prueba específica para valorar las fibras autonómicas (pequeñas no mielinizadas), las cuales no pueden evaluarse con los estudios convencionales. ${ }^{9}$ Sin embargo, al ser un procedimiento poco rutinario no fue posible replicar dicha prueba en la paciente. Para autores como Garay et. al, el cuadro clínico fue el principal sustento diagnóstico que se complementa con biopsia y ENMG. ${ }^{5}$

\section{Manifestaciones clínicas de las HSNA}

En relación a casos documentados con la misma patología Pacheco Cuéllar reportó dos familias mexicanas afectadas de NSAH IIA, donde se identificó una eliminación nueva de 8 nucleótidos c.1219 1226 de ITCTCAGCA, que ocasiona una proteína anormal. Los afectados presentaban ausencia de sensibilidad y amputaciones en las porciones distales de manos y pies, sin debilidad muscular ni manifestaciones autonómicas, PANS ausentes con edad de inicio entre la primera y la segunda décadas de la vida (9-20 años). 10

Mientras que C. Esmer y cols. reportaron en México un cuadro prácticamente idéntico en tres hermanos con alteración de la sensibilidad, coeficiente intelectual limítrofe, fuerza conservada, tono muscular disminuido, ninguna manifestación autonómica, reflejos ausentes y ausencia de los PANS que se correlacionan con el cuadro del caso clínico expuesto y sin antecedente familiar de neuropatía; sin embargo, a estos pacientes se les realizó el estudio molecular, identificando una mutación previamente reportada en un canadiense con ascendencia libanesa en la secuencia WNK12, lo que sugiere que en México exista población con ascendencia de este tipo y portadora de la patología o sea resultado de una nueva mutación que supera lo esperado en esta secuencia. ${ }^{2,10}$

Otros casos reportados con patrón HSNA tipo II presentan un marcado compromiso sensorial, mutilaciones en manos y pies, osteomielitis, fracturas frecuentes, 
deformidades en manos y pies, atrofia de papilas gustativas y afección a estructuras de cara, con funciones cognitivas normales o limítrofes. Datos que se correlacionan con la evolución y cuadro clínico de la paciente. ${ }^{2,6,8}$

\section{Problemas dermatológico y anexos}

Debido a las lesiones autoinflingidas que se caracterizan por tener un bajo porcentaje de curación y alta recurrencia, se convierten en motivos de consulta frecuente. ${ }^{5}$ Los problemas oculares como úlceras corneales suelen disminuir la agudeza visual y comprometer el nivel de independencia. En el caso de esta paciente, la persistencia de lesiones en cara y miembros inferiores a pesar de tratamiento farmacológico y cuidados de los familiares se originó por un componente conductual que llevó a la paciente a laceraciones constantes.

\section{Problemas ortopédicos y alteracio- nes de la marcha}

En las HSNA la alteración molecular en la función del factor de crecimiento neuronal (NGF) también afecta el proceso normal de consolidación de la fractura. El metabolismo óseo se ve afectado por la falta de fibras nociceptivas, presente no sólo en la piel, sino también en el sistema esquelético. ${ }^{11}$ Estos factores producen un mayor riesgo de fracturas y evolución tórpida como en el caso expuesto. La marcha atáxica está presente en la mayoría de las NSAH, con aumento en la base de sustentación, caídas frecuentes, además de ausencia de los reflejos osteotendinosos y requiriendo hasta en un $50 \%$ de los casos asistencia para la marcha, produciendo baja movilidad y estructuración de contracturas. ${ }^{1,12}$ Para este caso las contracturas ya estaban estructuradas, impidiendo la bipedestación requiriendo de ayuda para los traslados, mientras que las trans- ferencias las realizaba por su cuenta sin cuidar de la integridad de tejidos blandos.

\section{Problemas intelectuales y de conducta}

El desarrollo cognitivo es normal o limítrofe en la HSNA tipo II, lo que la diferencia de las tipos III y IV, donde se reporta discapacidad intelectual leve a moderada. ${ }^{2-4,8}$ Las alteraciones conductuales, ya reportadas en otros casos como trastornos obsesivo-compulsivos, ansiedad y problemas de aprendizaje suelen complicar su evolución si no se realiza una intervención temprana. ${ }^{13}$ En el caso de esta paciente se dio manejo por paidopsiquiatría a base de risperidona, con un fracaso en el tratamiento por falta de apego a las indicaciones.

\section{Otras manifestaciones clínicas en las HSNA}

Existen otras manifestaciones que también deben tomarse en cuenta sobre los aparatos cardiovascular, digestivo, respiratorio, renal y visual que causan grados variables de discapacidad y función, dependiendo del tipo de mutación. ${ }^{12}$

Durante el seguimiento de la paciente no se documentaron alteraciones cardiovasculares, como arritmias, hipotensión ortostática o cambios de la tensión arterial, datos que corresponden con las HSNA tipo II en comparación con la tipo III que presentan mayores manifestaciones autonómicas. ${ }^{14}$ En relación al apartado respiratorio, la paciente nunca presentó modificaciones en la saturación de $\mathrm{O}_{2}$ ni retención de $\mathrm{CO}_{2}$, manteniendo un adecuado patrón ventilatorio y sin episodios infecciosos, ni alteraciones respiratorias durante el sueño, los cuales se han documentado en mayor frecuencia en el tipo III. ${ }^{15}$ 


\section{Conclusiones}

Las HSAN deben sospecharse en pacientes con alteraciones del desarrollo psicomotor, tono muscular, reflejos disminuidos, problemas sensoriales, hipertermia u otras manifestaciones autonómicas. Es obligado valorar las vías sensoriales (dolor) en pacientes pediátricos y tener en cuenta que tiene una gran variabilidad en su presentación clínica.

Existen pocos casos reportados de este tipo de patologías. La comprensión de la fisiopatología es fundamental para brindar un tratamiento óptimo y medidas preventivas, ya que las complicaciones suelen causar una discapacidad múltiple y severa, así como un pobre pronóstico de vida. El exponer estos casos apoya en el conocimiento y diseño de estrategias para su tratamiento.

El estudio molecular sigue siendo el estándar de oro. Cuando estos son inaccesibles, los estudios de electroneuromiografía ofrecen un apoyo importante en el escrutinio y evaluación de las fibras nerviosas, ya que son altamente confiables para detectar neuropatías, sin requerir un estudio molecular o biopsia de primera instancia para iniciar una intervención.

\section{Bibliografía}

1. Vaughan $G$, Norcliffe-Kaufmann L, Löken L, Axelrod $F$, Kaufmann $H$. Disturbances in affective touch in hereditary sensory \& autonomic neuropathy type III. International Journal of Psychophysiology 93. 2014; 56-61.

2. Esmer C, Díaz Zambrano $S$, Santos Díaz $M A$ González Huerta LM , Cuevas Covarrubias SA, et al. Neuropatía sensitiva autonómica hereditaria tipo IIA: manifestaciones neurológicas y esqueléticas tempranas. An Pediatr (Barc). 2014; 80(4):254-258.

3. Axelrod F, Gold-von G. Hereditary sensory and autonomic neuropathies: types II, III, and IV. Orphanet Journal of Rare Diseases 2007, 2:39 doi:10.1186/1750-1172-2-39.

4. Albuja Echeverría B, Alvear Lozanoa MB, Ordóñez Paredesa.CP. Congenital insensitivity to pain with anhidrosis. Clinical diagnosis, evolution and complications. Case report. Arch Argent Pediatr 2014;112(5):e200-e205 / e200.

5. Garay M, Moreno S, Huczak, Aprea G. neuropátia sensitiva y autonomica hereditaria tipo II. Dermatol Pediatr Latinoam (En línea). 2013; 11 (3): 102-6

6. Annelies Rotthier, Jonathan Baets, Els De Vriendt, An Jacobs, Michaela Auer-Grumbach, Genes for hereditary sensory and autonomic neuropathies: a genotype-phenotype correlation. Brain 2009: 132; 2699-2711

7. Palma JP, Kaufmann L, Fuente C, Percival L, Mendoza $C$, et al. Current Treatments in Familial Dysautonomia. Expert Opin Pharmacother. 2014 December ; 15(18): 2653-2671.
8. Mobini M, Javadzadeh A, Forghanizadeh J. Neuropathic osteroarthropathy in a patient with congenital insensivity to pain. Arch Iran Med 2009;12(6):599 602

9. Kucera P, Goldenberg Z, Kurca E, Sympathetic skin response: review of the method and its clinical use, Bratist Lek Listy 2004;105(3):108-116.

10. Pacheco-Cuellar G, González-Huerta LM, ValdésMiranda JM, Peláez-González H, Zenteno-Bacheron $\mathrm{S}$, Cazarin-Barrientos J, et al. Hereditary sensory and autonomic neuropathy ii due to novel mutation in the HSN2 gene in Mexican families. J Neurol. 2011;258:1890---2.

11. Channa Maayan H. Respiratory aspects of Riley-Day Syndrome: Familial Dysautonomia. Paediatric respiratory reviews (2006) 7S, S258-S259

12. Macefield VG, Norcliffe-Kaufmann $\amalg$, Axelrod FB, et al. Relationship between proprioception at the knee joint and gait ataxia in HSAN III. Mov Disord. 2013 Jun; 28(6):823-7. [PubMed: 23681701]

13. Sands SA, Giarraffa $P$, Jacobson $C M$, et al. Familial dysautonomia's impact on quality of life in childhood, adolescence, and adulthood. Acta Paediatr. 2006 Apr; 95(4):457-62. [PubMed: 16720494]

14. Norcliffe-Kaufmann L, Axelrod F, Kaufmann H. Afferent baroreflex failure in familial dysautonomia. Neurology 2010;75(21):1904-11.

15. Pérez-López, Cabrera-González, Gutiérrez-de la Iglesia, Knörr-Giménez. Update Review and Clinical Presentation in Congenital Insensitivity to Pain and Anhidrosis. Case Rep Pediatr. 2015; 2015: 589852 


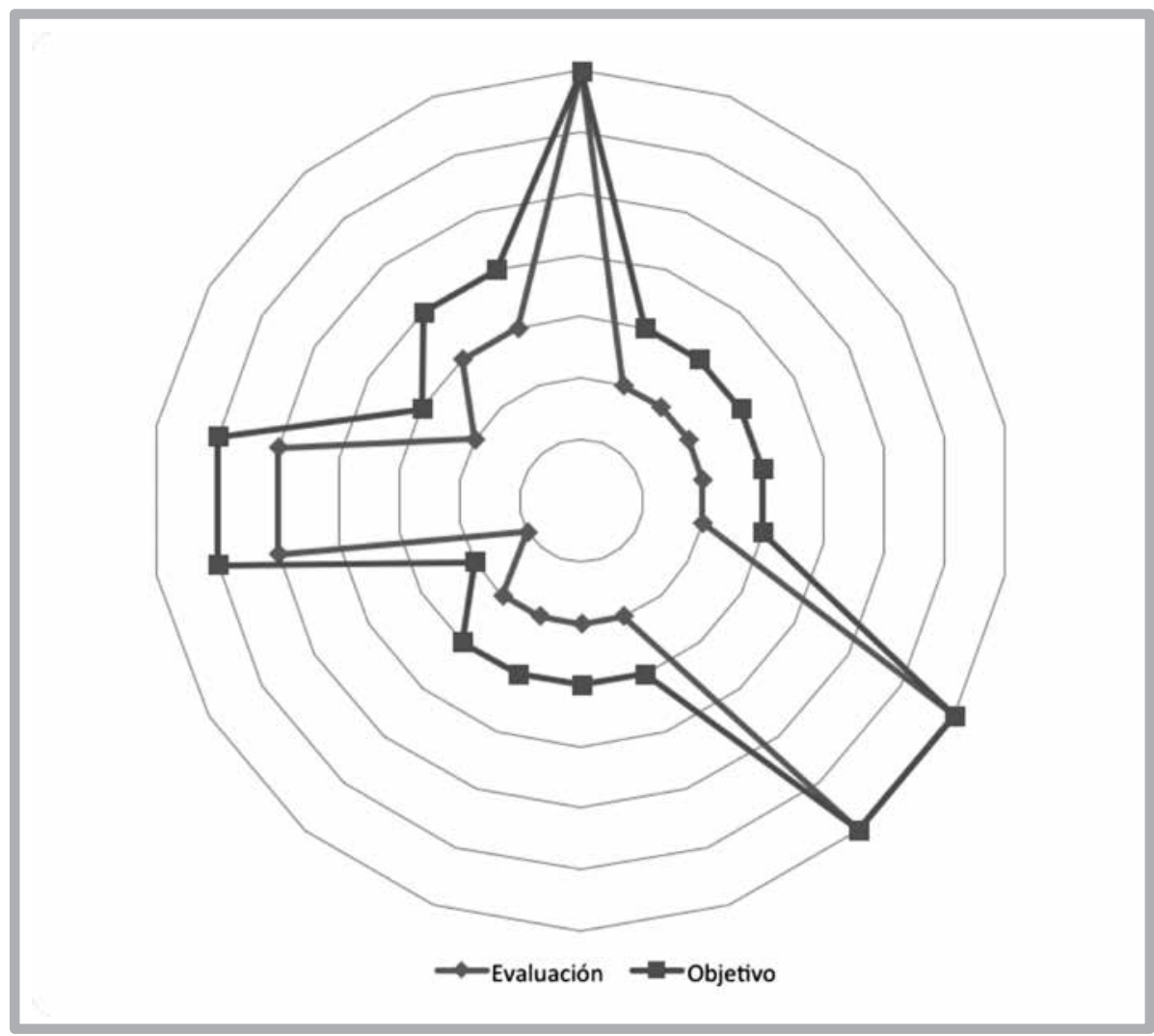

Figura 1.- Evaluación inicial de Wee-Fim que muestra un mayor impacto en las áreas de movilidad, derivados de la HSNA.

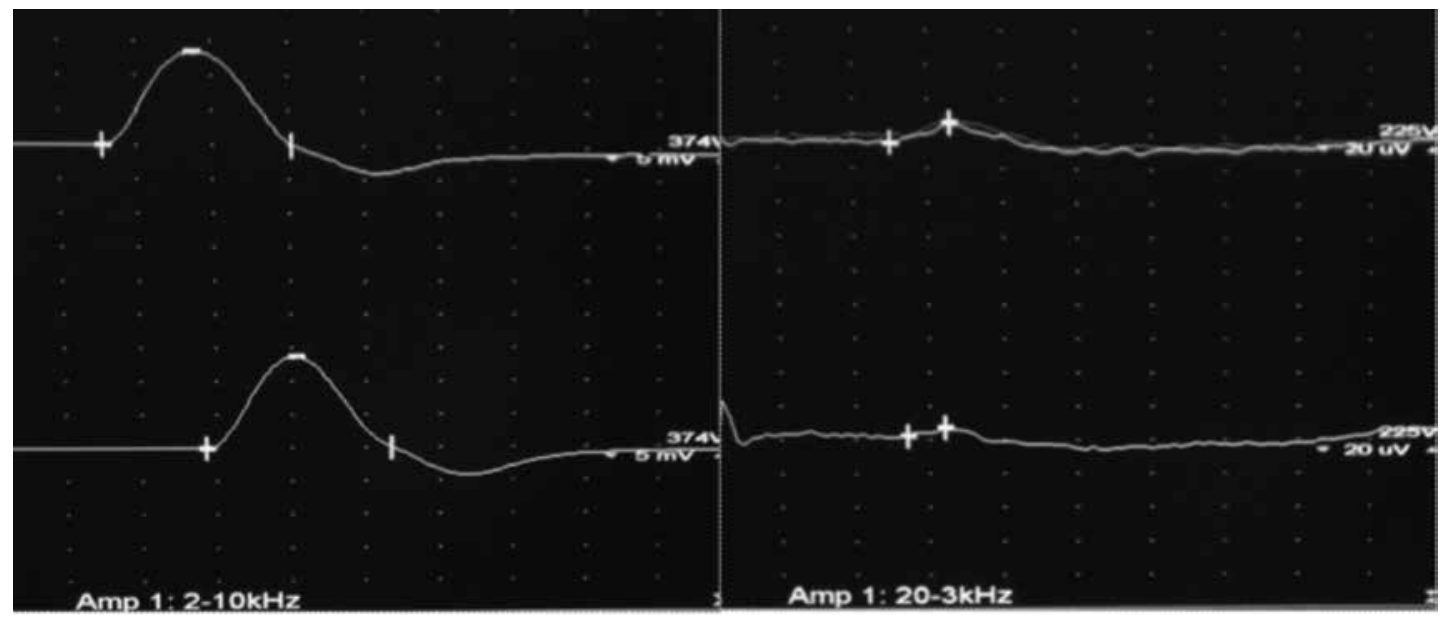

Figura 2.- Comparación de las respuesta motoras vs sensoriales. Nótese la disminución de la amplitud de los potenciales de acción nervioso sensoriales (PANS). 


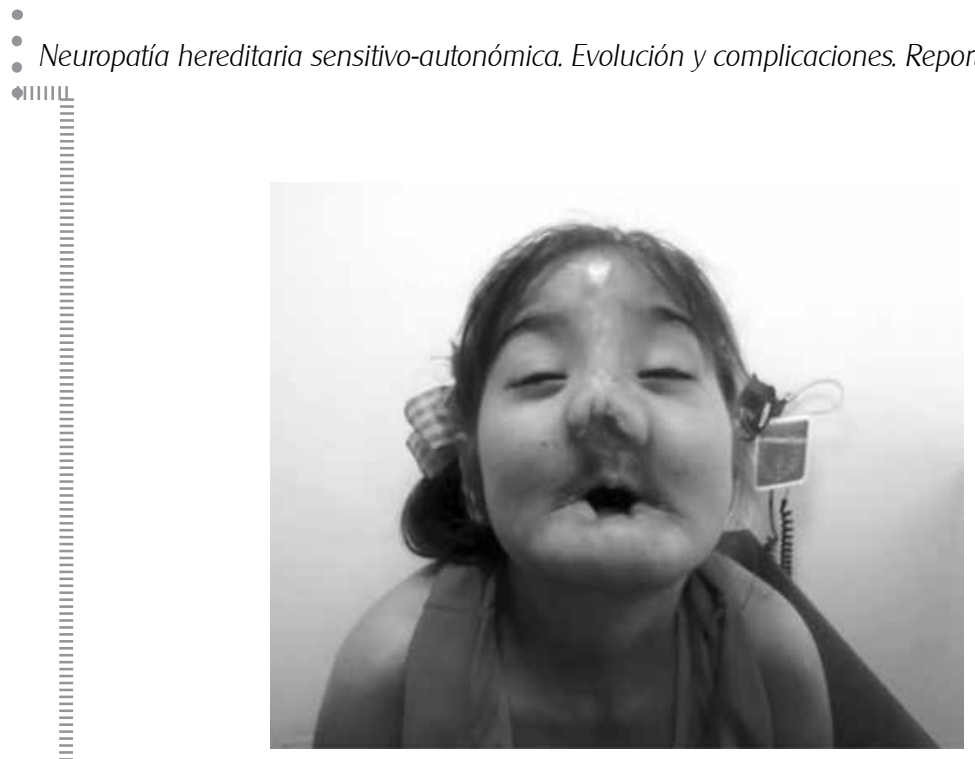

Arriaga-Rivera Javier et al

Imagen 1.- Muestra las alteraciones facial, con mutilaciones a nivel nasal y adoncia.

Imagen 2.- Nótese las amputaciones distales de falanges en ambas manos.
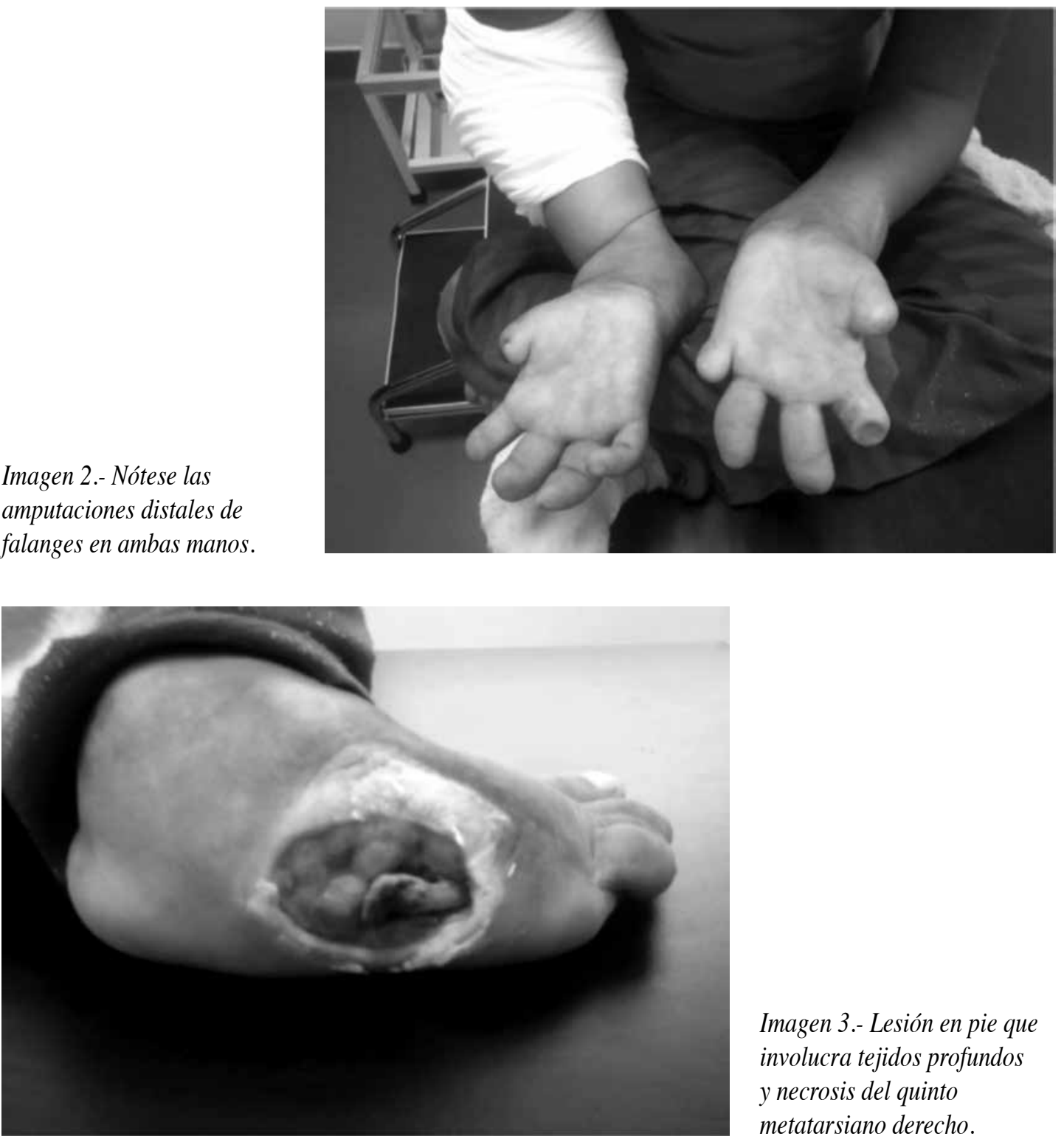

Imagen 3.- Lesión en pie que involucra tejidos profundos y necrosis del quinto metatarsiano derecho. 


\section{Tabla I}

\section{Clasificación de Erlanger-Gasser y numérica de las fibras nerviosas.}

\begin{tabular}{|c|c|c|c|c|c|}
\hline Tipo & Numero & Función & Tamaño (nm) & Mielina & Velocidad de conducción $\mathrm{m} / \mathrm{s}$ \\
\hline A Alfa & la & $\begin{array}{l}\text { Propiocepción, } \\
\text { estiramiento,(husos muscula- } \\
\text { res-receptores anulo espina- } \\
\text { les) y motoras extrafusales }\end{array}$ & 17 & + & $70-120$ \\
\hline & Ib & $\begin{array}{l}\text { Fuerza contráctil (órgano } \\
\text { tendinoso de Golgi) }\end{array}$ & 16 & + & $70-120$ \\
\hline A Beta & II & $\begin{array}{l}\text { Presión, estiramiento (huso } \\
\text { muscular), tacto, vibración. }\end{array}$ & 8 & + & $30-70$ \\
\hline A Gamma & II & Fibromusculares intrafusales & $2-8$ & + & $15-30$ \\
\hline A Delta & III & Dolor, temperatura, tacto & $1-5$ & + & $5-30$ \\
\hline B & & $\begin{array}{l}\text { Axones pre ganglionares } \\
\text { simpáticos }\end{array}$ & $<3$ & + & $3-15$ \\
\hline C & IV & $\begin{array}{l}\text { Dolor, temperatura, axones } \\
\text { pos ganglionares }\end{array}$ & $0.1-0.3$ & - & $0.6-2$ \\
\hline
\end{tabular}

\section{Tabla 2}

\section{Clasificación y características de las neuropatías hereditarias sensitivo-autonómicas.}

\begin{tabular}{|c|c|c|c|c|c|}
\hline Características & HSAN tipo I & HSAN tipo II & $\begin{array}{l}\text { HSAN tipo III. } \\
\text { Disautonomía } \\
\text { familiar/Síndrome } \\
\text { de Riley Day }\end{array}$ & $\begin{array}{l}\text { HSAN tipo IV. } \\
\text { Inestabilidad } \\
\text { congénita al dolor } \\
\text { con anhidrosis }\end{array}$ & HSAN tipo $\mathrm{V}$ \\
\hline Herencia & $A D-A R$ & $A R$ & $\mathrm{AR}$ & $\mathrm{AR}$ & $\mathrm{AR}$ \\
\hline Gen & $\begin{array}{c}\text { SPTLC1, } \\
\text { RAB7, ATL1 }\end{array}$ & $\begin{array}{c}\text { WNK1, FAM134B } \\
\text {, KIF1A }\end{array}$ & IKBKAP & NTRK1 & NGFB \\
\hline Edad de inicio & 2a década de vida & $\begin{array}{c}\text { Congénita, } \\
\text { Infancia temprana }\end{array}$ & Nacimiento & Nacimiento & Nacimiento \\
\hline Anestesia & ++ & ++ & + & ++ & ++ \\
\hline Hidrosis & Normal & $\begin{array}{c}\text { Normal/disminuida } \\
\text { en parches }\end{array}$ & Disminuida & Disminuida & Normal \\
\hline $\begin{array}{l}\text { Discapacidad } \\
\text { intelectual } \\
\end{array}$ & Normal & Limítrofe/Normal & Leve & Moderado & Limítrofe \\
\hline Tono muscular & Disminuido & Dismunuido & Disminuido & Dismunuido & Dismunuido \\
\hline $\begin{array}{l}\text { Reflejos } \\
\text { osteotendinosos }\end{array}$ & Ausente & Ausentes & Ausentes & $\begin{array}{c}\text { Normal o } \\
\text { disminuidos }\end{array}$ & Normal \\
\hline PAMC & Variable & $\begin{array}{l}\text { Normales o } \\
\text { disminuidos }\end{array}$ & Variable & Variable & Normal \\
\hline PANS & $\begin{array}{l}\text { Disminuido } \\
\text { o ausente }\end{array}$ & $\begin{array}{l}\text { Disminuido } \\
\text { o ausente }\end{array}$ & $\begin{array}{l}\text { Disminuido } \\
\text { o ausente }\end{array}$ & $\begin{array}{l}\text { Disminuido } \\
\text { o ausente }\end{array}$ & Normal \\
\hline $\begin{array}{l}\text { Hallazgos } \\
\text { en biopsia }\end{array}$ & $\begin{array}{l}\text { disminución } \\
\text { de FA y FM }\end{array}$ & FM ausente & Reducción FA & Ausencia FA & $\begin{array}{l}\text { Ausencia de } \\
\text { FM pequeñas }\end{array}$ \\
\hline
\end{tabular}

Tomado de Mobini M (2009) y modificado.

$A D$ : autosómico dominante.

AR: autosómico recesivo.

FA: fibras amielínicas
FM: fibras mielinizadas.

PAMC: potencial de acción muscular compuesto.

PANS: potencial de acción nerviosos sensorial. 